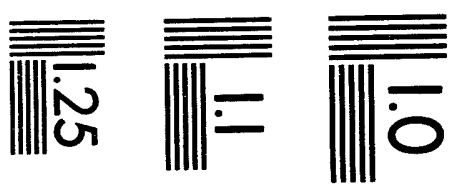

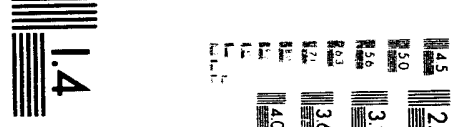

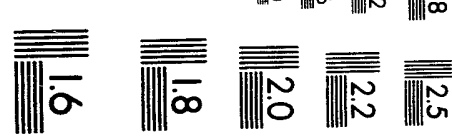



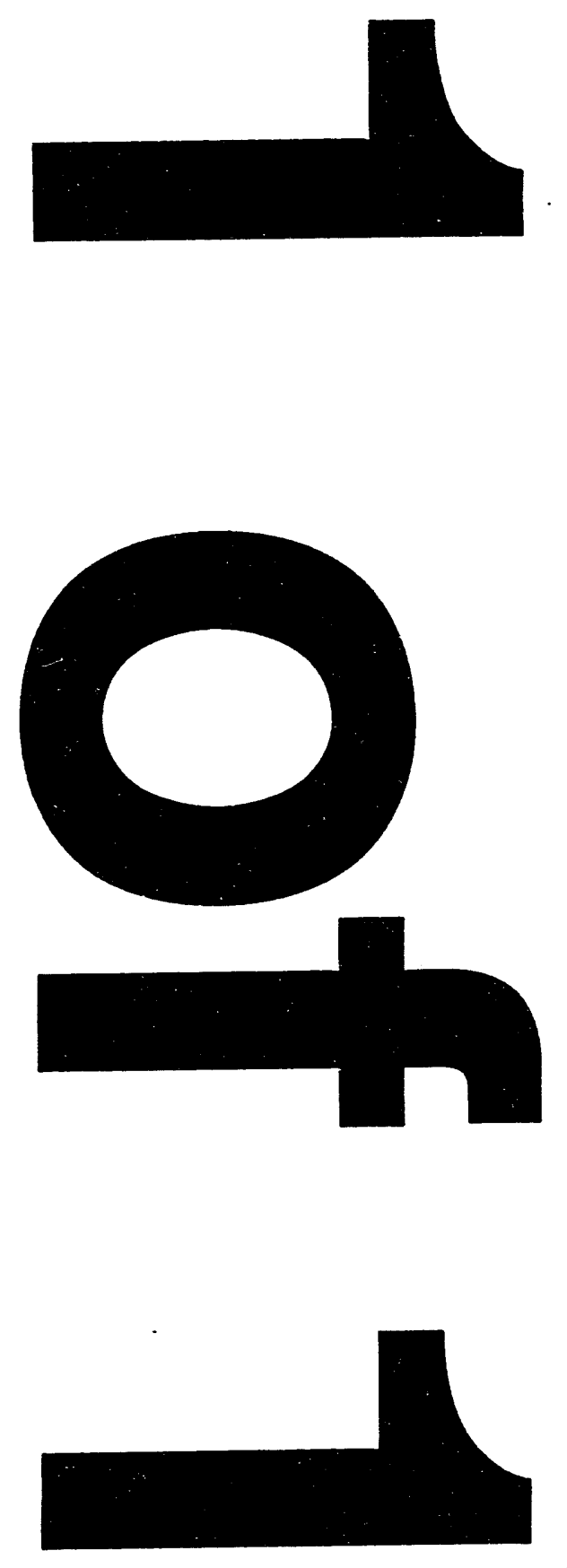


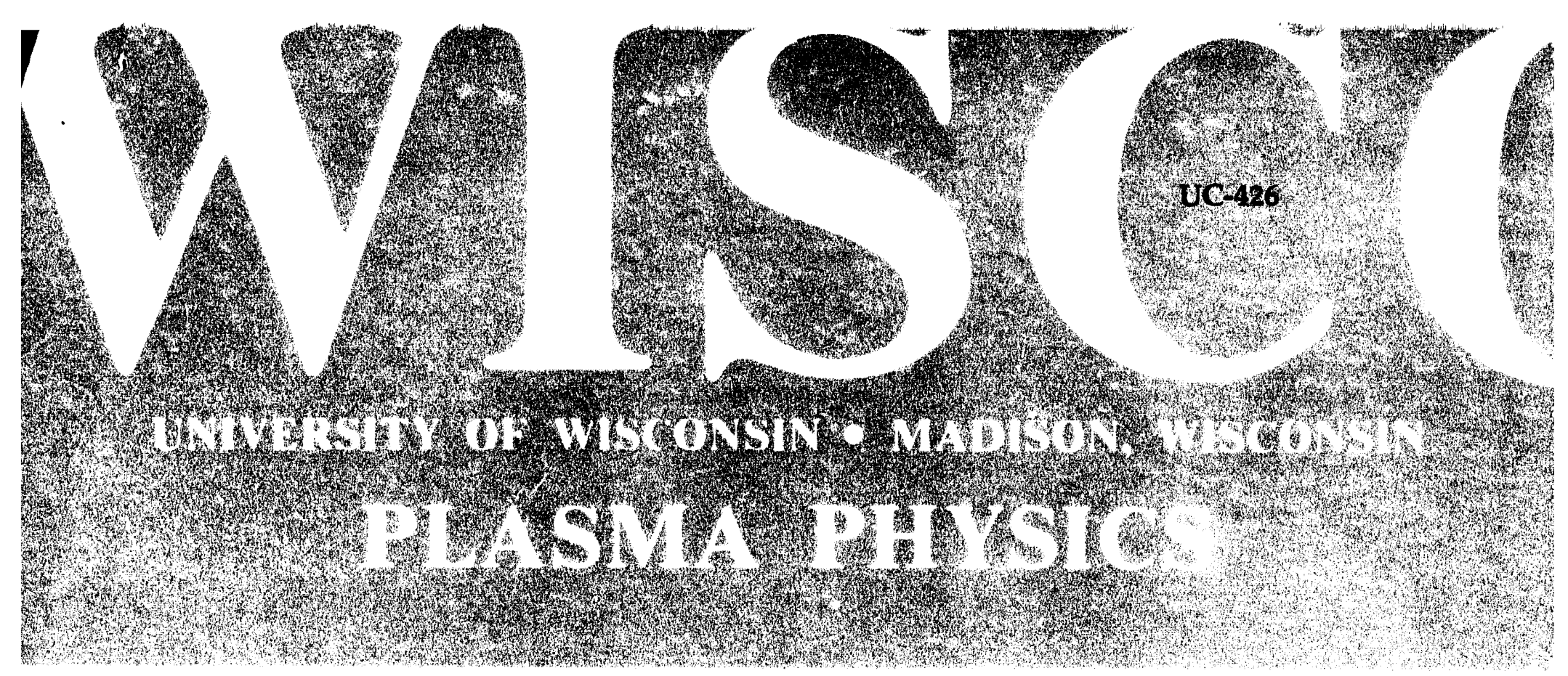

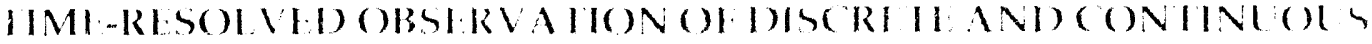

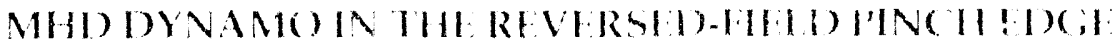

H. Ji, M.F. Almagri, S.C. Prager, J.S. Sarff

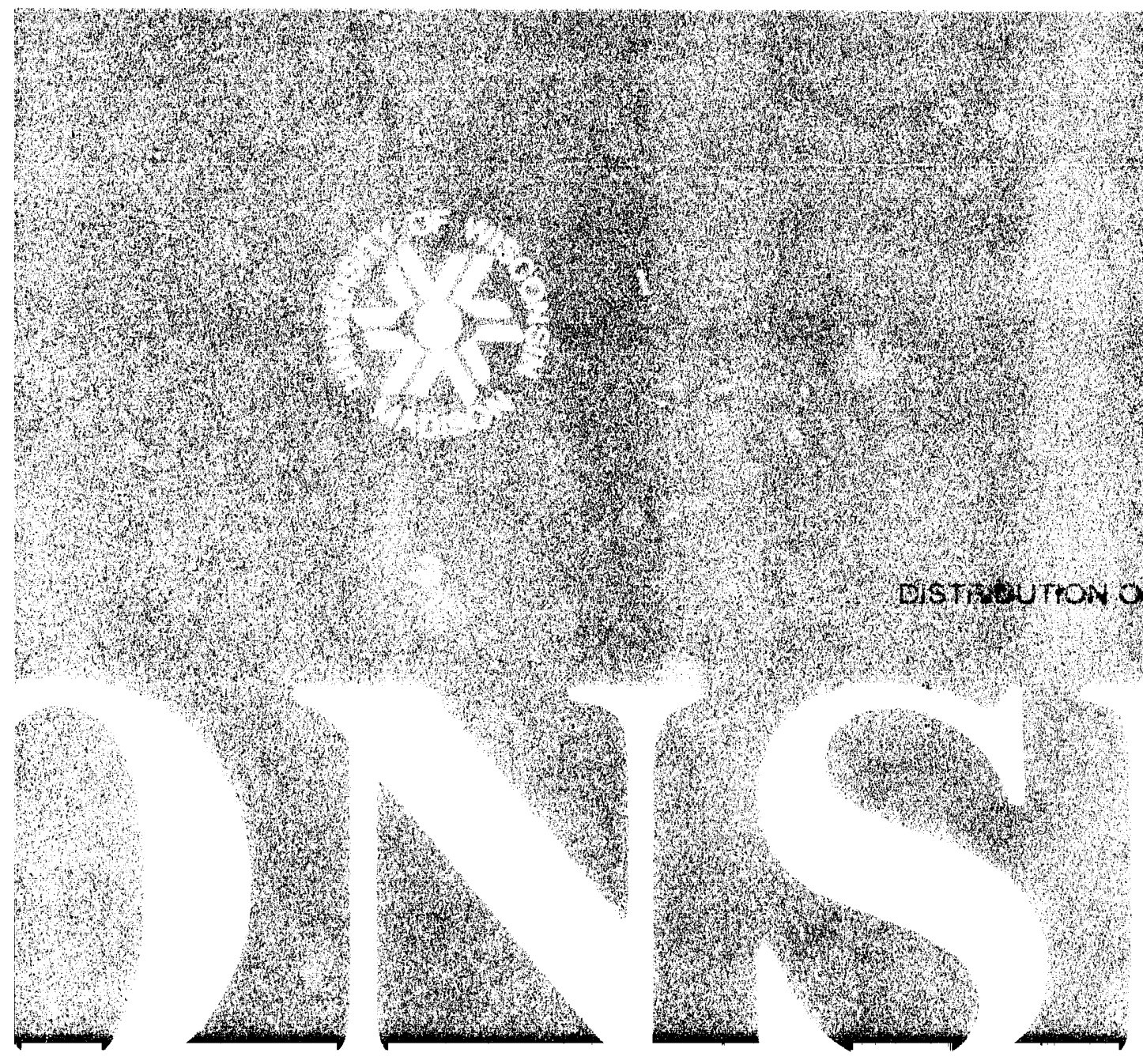
3
7
7
7

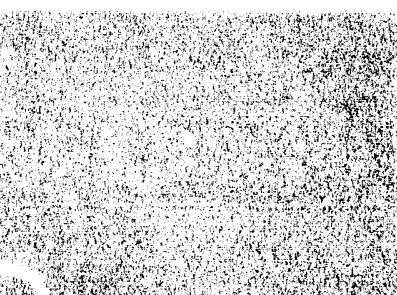
+1.7x 7
7
7

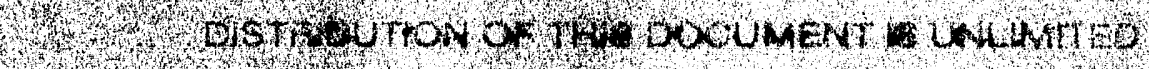

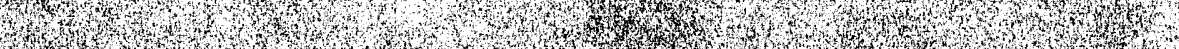

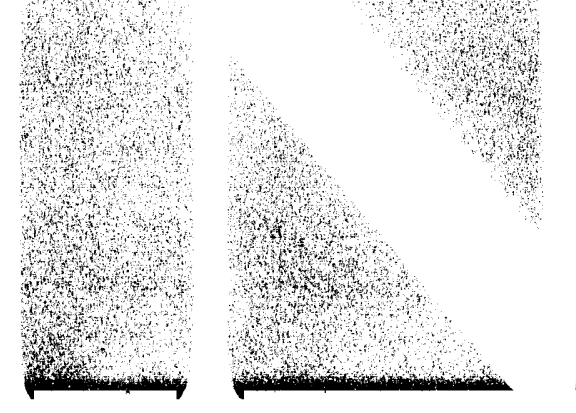




\section{NOTICE}

This report was prepared as an account of work sponsored by an agency of the United States Government. Neither the United States nor any agency thereof, nor any of their employees, makes any warranty, expressed or implied, or assumes any legal liability or responsibility for any third party's use or the results of such use of any information, apparatus, product or process disclosed in this report, or represents that its use by such third party would not infringe privately owned rights.

Printed in the United States of America Available from

National Technical Information Service

U.S. Department of Commerce 5285 Port Royal Road Springfield, VA 22161

NTIS Price codes

Printed copy: $\quad$ A02

Microfiche copy: A01 


\title{
Time-resolved Observation of Discrete and Continuous MHD Dynamo in the Reversed-Field Pinch Edge
}

\author{
H. Ji, A.F.Almagri, S.C.Prager, and J.S.Sarff \\ Department of Physics, University of Wisconsin, Madison, Wisconsin 53706 \\ (January 6, 1994)
}

\begin{abstract}
We report the first experimental verification of the MHD dynamo in the RFP. A burst of magnetohydrodynamic (MHD) dynamo electric field is observed during the sawtooth crash, followed by an increase in the local parallel current in the MST RFP edge. By measuring each term, the parallel MHD mean-field Ohm's law is observed to hold within experimental error bars both between and during sawtooth crashes.
\end{abstract}

PACS numbers: $52.55 . \mathrm{Hc}, 52.25 . \mathrm{Gj}, 52.35 . \mathrm{Ra}$

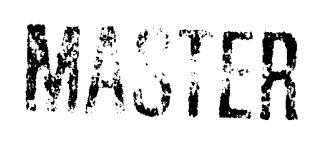

Typeset using REVTEX 
The dynamo phenomenon, in which the magnetic-field-aligned electric current is selfgenerated by plasma dynamics, has been a mystery in magnetically confined laboratory plasmas and astrophysical plasmas for many decades. The reversed-field-pinch (RFP) toroidal plasma, in which the toroidal field reverses its direction at the edge, is a particularly vivid example of the dynamo effect. In the RFP, the externally applied electric field is in the toroidal direction. Thus, the poloidal current near the edge, essentially parallel to the magnetic field, is generated and maintained by the dynamo. In the widely studied MHD dynamo model, a fluctuation-induced electromotive electric field $\langle\widetilde{\boldsymbol{v}} \times \widetilde{\boldsymbol{B}}\rangle_{\|}$sustains the field-aligned current against resistive decay in the parallel Ohm's law [1]

$$
E_{\|}+<\widetilde{\boldsymbol{v}} \times \widetilde{\boldsymbol{B}}>_{\|}=\eta j_{\|}
$$

where $E_{\|}$is the equilibrium electric field parallel to the magnetic field, $\eta$ the electric resislivity, $j_{\|}$the parallel equilibrium current, $\tilde{\boldsymbol{v}}$ and $\widetilde{\boldsymbol{B}}$ are the fluctuating fluid velocity and magnetic field respectively, and $\langle\ldots\rangle$ denotes an average over a flux surface. On the other hand, the kinetic dynamo theory (KDT) $[2,3]$ assumes that the dynamo is driven by radial diffusion of the parallel current due to a prescribed stochastic magnetic field. The KDT model is supported [4] by the existence of fast electrons detected at the edge with a temperature comparable to core electrons. These fast electrons carry most of the edge parallel current $[5,6]$, but the current diffusion hypothesized for their existence has never been directly measured.

The MHD model has been intensively investigated by a large number of authors through nonlinear computation [7], and it agrees fairly well with experimental tearing mode spectra and their nonlinear mode interactions. However, measurements in the REPUTE-1 RFP edge have shown [8] that the MHD dynamo electric field is not sufficient to account for $\eta j_{\|}-E_{\|}$. Contrariwise, recent measurements in a spheromak [9] indicate nonzero MHD dynamo electric fields.

In this Letter, we report a first experimental verification of the MHD dynamo in the RFP edge. In the Madison Symmetric Torus (MST) RFP an additional test of the dynamo is 
allowed by the presence of clear sawtooth oscillations [10]. The sawtooth crash is a discrete dynamo event during which substantial toroidal flux (and edge poloidal current) is generated. We observe the MHD dynamo electric field both as a burst during the sawtooth crash and continuously between the crashes, sufficient to sustain the parallel current. The difference in dynamo mechanisms active in MST and REPUTE is likely due to the substantially different edge conditions, as discussed later.

In MS' and the earlier experiments the MHD dynamo term is inferred from probe. measurement of the fluctuating electric field ( $\tilde{\boldsymbol{v}}$ is not measured directly). To interpret the measured quantities, we consider the generalized Ohm's law [11],

$$
-\frac{m_{e}}{e^{2} n} \frac{\partial \boldsymbol{j}}{\partial t}+\boldsymbol{E}+\boldsymbol{v} \times \boldsymbol{B}-\frac{1}{e n} \boldsymbol{j} \times \boldsymbol{B}+\frac{\boldsymbol{\nabla} P_{e}}{e n}=\eta \boldsymbol{j}
$$

where $n$ is the electron density and $P_{c}$ the electron pressure. By splitting every quantity into mean (denoted by subscript 0) and fluctuating (denoted by tildes) parts, averaging over a flux surface, and taking the parallel component, the parallel Ohm's law in a turbulent plasma becomes

$$
\eta_{\|} j_{\| 0}-E_{\| 0}=<\widetilde{\boldsymbol{v}} \times \widetilde{\boldsymbol{B}}>_{\|}-<\tilde{\boldsymbol{j}} \times \widetilde{\boldsymbol{B}}>_{\|} / e n,
$$

where we have neglected two small $\partial j_{\|} / \partial t$ and $\left\langle\tilde{\eta} \widetilde{j}_{\|}>\right.$terms, as appropriate for the experimental condition. The right-hand side includes the usual $<\widetilde{\boldsymbol{v}} \times \widetilde{\boldsymbol{B}}>$ term and the Hall term previously measured to be small in MST [12].

An alternative form of the parallel Ohm's law can be derived by substituting the perpendicuiar component of Eq.(1), $\widetilde{\boldsymbol{v}}_{\perp}-\widetilde{\boldsymbol{j}}_{\perp} / e n \approx\left(\widetilde{\boldsymbol{E}}_{\perp} \times \boldsymbol{B}_{0}+\boldsymbol{\nabla}_{\perp} \tilde{P}_{e} \times \boldsymbol{B}_{0} /\right.$ en $) / B^{2}$, into Eq.(2) to yield

$$
\eta_{\| j_{\| 0}}-E_{\| 0}=<\widetilde{\boldsymbol{E}}_{\perp} \cdot \widetilde{\boldsymbol{b}}_{\perp}>+<\nabla_{\perp} \widetilde{P}_{e} \cdot \widetilde{\boldsymbol{b}}_{\perp}>/ \text { en }
$$

where $\boldsymbol{b} \equiv \boldsymbol{B} / \boldsymbol{B}$. For simplicity of presentation, we have ignored the density fluctuations $\tilde{n}$, which would add a term $<\tilde{n} \nabla_{\|} \tilde{P}_{e}>/ e n^{2}$ in both Eq.(2) and Eq.(3), but would not change our conclusions. 
Interestingly, note that the usual $<\widetilde{\boldsymbol{v}} \times \widetilde{\boldsymbol{B}}>$ term consists of both $<\widetilde{\boldsymbol{E}}_{\perp} \cdot \widetilde{\boldsymbol{b}}_{\perp}>$ and $<\nabla_{\perp} \widetilde{P}_{\boldsymbol{e}} \quad \tilde{b}_{\perp}>/$ en terms, by comparing Eq.(2) and Eq.(3). The first term represents the contribution to $\tilde{\boldsymbol{v}}$ from the fluctuating $\boldsymbol{E} \times \boldsymbol{B}$ drift, while the second term is the contribution from the fluctuating diamagnetic drift. The latter one, which is $\left.\propto \partial<\widetilde{P}_{c} \tilde{b}_{r}\right\rangle / \partial r$ in the shearless limit, represents current diffusion in space due to $\tilde{b}_{r}$ and includes the KD'T mechanism. However, this term is not included in the pressureless MHD computations [7]. Thercfore, we identify the first term $\left\langle\widetilde{\boldsymbol{E}}_{\perp} \cdot \widetilde{\boldsymbol{b}}_{\perp}>\right.$ as 'the MHD dynamo term ', usually referred to as $\left\langle\tilde{\boldsymbol{v}} \times \widetilde{\boldsymbol{B}}>\right.$. The experiments described here are aimed to measure $<\widetilde{\boldsymbol{E}}_{\perp} \cdot \widetilde{\boldsymbol{b}}_{\perp}>$ which has two large components, $\left\langle\widetilde{E}_{t} \tilde{b}_{t}\right\rangle$ and $\left\langle\widetilde{E}_{r} \widetilde{b}_{r}\right\rangle$, since $B_{p} \gg B_{t}$ in RFP edge.

The MST [13] is a large $\mathrm{RFP}$ device with major radius of $1.50 \mathrm{~m}$, minor radius of $0.52 \mathrm{~m}$, and plasma, current up to $700 \mathrm{kA}$. The experiments reported here were carried out at the relatively low plasma current of $\simeq 210 \mathrm{kA}$ to avoid heat damage to the inserted probe and at sufficient density (chord-averaged density $\overline{n_{e}} \simeq 1.0 \times 10^{19} / \mathrm{m}^{3}$ ) to minimize disturbance of the triple probe measurement by the fast electrons. Density scan experiments have shown that the fast electron population [6] and its influence on probe measurement are significant only at low density $\left(n_{e}<0.8 \times 10^{19} / \mathrm{m}^{3}\right)$. All measurements presented here are taken during $t=13-25 \mathrm{~ms}$ around the current flattop.

Two versions of a complex probe [14] have been constructed to measure $\left\langle\widetilde{\boldsymbol{E}}_{\perp} \cdot \tilde{\boldsymbol{b}}_{\perp}\right\rangle$. Each version consists of two triple probes to measure electron temperature $T_{e}$, density $n$, and floating potential $V_{f}$ at two locations separated by $1.27 \mathrm{~cm}$ toroidally or $0.25 \mathrm{~cm}$ radially. The electrostatic components of electric fields $E_{t}$ and $E_{r}$ can be obtained from the difference in plasma potential $V_{p}=V_{f}+c T_{e}$ where $c$ is a constant. (The inductive components are negligible in MST.) Magnetic pick-1p coils for $B_{t}$ and $B_{r}$ are also installed to infer $\left\langle\tilde{E}_{t} \widetilde{b}_{t}\right\rangle$ and $\left\langle\tilde{E}_{r} \tilde{b}_{r}>\right.$. A separate, small, insertable Rogowskii coil probe [15] measures the local poloidal (parallel) current.

The measurements at each radial position were carried out in 30 identical discharges with 150 sawtooth crashes. To obtain ensemble-averaged quantities (such as cross correlations) 
with time-resolved information during a sawtooth crash, an ensemble is constructed from time samples time-referenced to a crash instead of the conventional cross-spectra method for stationary turbulence. Since the plasma rotates in the laboratory frame, this method is equivalent to flux surface averaging even though the measurement position is fixed. Using phase-shifted fluctuations calculated via the Fast Fourier Transform, we calculate the timeresolved coherence $\gamma$ and phase shift $\theta$ between two fluctuating quantities.

Sawtooth oscillations in MST represent discrete dynamo events. Figure 1 illustrates field generation and relaxation over two sawtooth oscillations spanning 5ms. Strong spontaneous field generation is evident in the sudden increase of the toroidal flux $\Phi_{t}$ during a sawtooth crash (in $\sim 0.1 \mathrm{~ms}$ ). Between crashes, flux generation (opposing resistive decay) is present but mild. The decreases in the pinch parameter $\Theta \equiv B_{p}(a) /\left(\Phi_{t} / \pi a^{2}\right)$ and the reversal parameter $F \equiv B_{t}(a) /\left(\Phi_{t} / \pi a^{2}\right)$ show that the plasma relaxes toward the minimum energy state with a flatter current profile, i.e., current decreases at the core and increases at the edge. The edge density and electron temperature also increase during a crash, as represented in Fig. 1 by the ion saturation current $J_{\text {sat }}$ measured by the triple probe at $r / a=0.98$. The time derivative of the flux, measurable as the voltage across the toroidal gap in the shell, $V_{\mathrm{tg}}$, is employed as a trigger for the sawtooth ensemble averaging and as a time reference throughout the paper.

Ensemble-averaged fluctuation amplitudes, coherence, and phase shift during one sawtooth, measured at $5 \mathrm{~cm}$ from the wall $(r / a=0.90)$, are shown in Fig.2. Fluctuation amplitudes peak at the sawtooth crash (except for $\left.\left|\widetilde{E}_{t}\right|\right)$, while the coherence is low $(\simeq 0.1)$ and the phase shift is almost $\pi$ (anti-phase) between $\widetilde{\boldsymbol{E}}_{\perp}$ and $\widetilde{\boldsymbol{B}}_{\perp} \cdot\left(\gamma_{E_{\mathrm{t}} B_{t}}\right.$ and $\theta_{E_{t} B_{\mathrm{t}}}$ are not shown, but they are similar to their counterparts of $<\widetilde{E}_{r} \tilde{b}_{r}>$.)

The two components of $\left\langle\widetilde{\boldsymbol{E}}_{\perp} \cdot \widetilde{\boldsymbol{b}}_{\perp}\right\rangle$ measured at $r / a=0.90$ are shown in Fig.3. Both $<\widetilde{E}_{t} \tilde{b}_{t}>$ and $<\widetilde{E}_{r} \tilde{b}_{r}>$ peak during the crash. The local poloidal current density $j_{p}$ keeps rising during the crash and peaks at the end of the crash, consistent with current profile flattening.

To establish the strength of the MHD dynamo term we compare it to other measured 
terms in Ohm's law (Eq.(3)). In Fig.4(a), we compare the measured MHD dynamo electric field to the resistive term $\eta j_{\|}$, where $\eta$ is Spitzer's resistivity calculated from the measured local $T_{e}$ but estimated $Z_{\text {eff }}=3$. In spite of large experimental error bars, fairly good agreement can be seen between $\left\langle\widetilde{\boldsymbol{E}}_{\perp} \cdot \widetilde{\boldsymbol{b}}_{\perp}\right\rangle$ and $\eta j_{\|}$except for the burst of dynamo clectric field during the crash. The electric field term is included in Fig.4(b) which compares $<\widetilde{\boldsymbol{E}}_{\perp} \cdot \widetilde{\boldsymbol{b}}_{\perp}>$ to $\eta j_{\|}-E_{\|}$. The parallel electric field at the edge is given by

$$
E_{\|}(r) \approx\left(V_{\mathrm{tg}}-2 \pi \int_{r}^{a} \dot{B}_{t} r \mathrm{~d} r\right) / 2 \pi r
$$

where the first term dominates. By including the electric field, Fig.4(b) shows good agreement between $<\widetilde{\boldsymbol{E}}_{\perp} \cdot \widetilde{\boldsymbol{b}}_{\perp}>$ and $\eta j_{\|}-E_{\|}$at all times.

The emerging physical picture of MST edge dynamo can be separated into two stages: (a) a continuous dynamo electric field drives equilibrium poloidal current between the sawtooth crashes and (b) the crash generates a burst of (discrete) dynamo electric field which is largely balanced by an inductive $E_{\|}$during the $j_{\|}$rising phase. The effective inductance (an be estimated as $l=E_{\|} /\left(\mathrm{d} j_{\|} / \mathrm{d} t\right) \sim 6 \times 10^{-9} \mathrm{Hm}$. The resulting $\mathrm{L} / \mathrm{R}$ time constant is $\tau=l / \eta \sim 0.2 \mathrm{~ms}$, consistent with the decay time of $j_{\|}$after the sawteeth.

The radial profiles of $\left\langle\widetilde{\boldsymbol{E}}_{\perp} \cdot \widetilde{\boldsymbol{b}}_{\perp}\right\rangle$ and $\eta j_{\|}-E_{\|}$are shown in Fig.5. The parallel MHD Ohm's law (Eq.(3) without the second term in the right-hand side) holds both between and during the sawtooth events within the experimental error bars. This result provides exprimental verification of MHD dynamo hypothesis in the RFP.

The MST result is in contrast, with the first such measurement [8] performed in the REPUTE-1 RFP edge, in which the MHD dynamo electric field is not sufficient to account for $\eta j_{\|}-E_{\|}$. However, we notice that the edge plasma is distinctly different in the two devices in two properties. First, the edge magnetic field in REPUTE is likely more stochastic than in MST. REPUTE experiences resistive wall instabilities since its shell penetration time is short ( $1 \mathrm{~ms}$ versus $>100 \mathrm{~ms}$ in MST). REPUTE also has larger field errors arising from larger ports and toroidal field ripple. Second, the REPUTE edge is collisional $\left(n \sim 2 \times 10^{19} \mathrm{~m}^{-3}\right.$, $T_{e} \sim 10 \mathrm{eV}$, electron mean free path $\lambda_{e} \sim$ several $\mathrm{cm}$ ), whereas the MST edge is relatively 
collisionless $\left(n \sim 2 \times 10^{18} \mathrm{~m}^{-3}, T_{e} \sim 30 \mathrm{eV}, \lambda_{e} \sim 2 \mathrm{~m}\right)$.

These differences suggest that REPUTE is more prone to a kinetic dynamo mechanism. Electron current can diffuse radially by following the stochastic field generated from slowly growing resistive wall instabilities or dc field errors. A collisionless model which incorporates self-consistency constraints (i.e. the effect of electron motion on the fluctuations through Ampere's Law) predicts negligible current diffusion by the KDT mechanism [16]. However, the constraints may not apply to the collisional REPUTE plasma, particularly if the fluctuations are partly external (i.e. due to field errors); thus a finite electron momentum transport may be possible as in the KDT model.

In conclusion, time-resolved measurements of discrete and continuous MHD dynamo have been performed in MST RFP edge. The observed MHD dynamo electric field is sufficient to sustain the parallel current. We speculate that observed different dynamo mechanisms in different machines depend upon the edge conditions, such as the presence of field errors or the collisionality. A comprehensive physical picture of the dynamo phenomena requires measurement of the MHD dynamo electric field under varying plasma conditions, and direct measurement of current diffusion (e.g. $<\tilde{P}_{\|}^{e} \tilde{b}_{r}>$ ).

The authors are grateful to MST group members, particularly Dr. S. Hokin, Dr. W. Shen and M. Stoneking, for their experimental contributions, and to Prof. P. Terry and Prof. Z. Yoshida for valuable discussions. This work was supported by the U.S. Department of Energy. 


\section{REFERENCES}

[1] C.G. Gimblett and M.L. Watkins, in Proceedings of the 7th European Conference on Controlled Fusion and Plasma Physics, Lausanne, 1975 (European Physical Society, Geneva, 1975), Vol.1, p.103.

[2] A.R. Jacobson and R.W. Moses, Phys. Rev. A, 29, 3335(1984).

[3] R.W. Mosce, K.F. Schocnberg and D.A. Baker, Phys. Fluids, 31, 3:52(1988).

[4] K.F. Schoenberg and R.W. Moses, Phys. Fluids, B3, 1467(1991).

[5] J.C. Ingraham et al., Phys. Fluids, B2, 143(1990).

[6] M.R. Stoneking et al., Bull. Am. Phys. Soc., 38, 1978(1993).

[7] For example, the earliest simulation result is E.J. Caramana, R.A. Nebel and D.D. Schnack, Phys. Fluids, 26, 1305(1983); and the newest one is A. Nagata et al., Phys. Fluids, B5, 1263(1993).

[8] H. Ji el al., Phys. Rev. Lett. 69, 616(1992).

[9] A. al-Karkhy et al., Phys. Rev. Lett. 70, 1814(1993).

[10] S. Hokin, et al., Phys. Fluids, B3, 2241(1991).

[11] L. Spitzer, Jr., Physics of Fully Ionized Ciases (2nd Revised Edition), (Interscience Publishers, New York, 1962), p.28.

[12] W. Shen and S.C. Prager, Phys. Fluids, B7, 1931(1993).

[13] R.N. Dexter et al., Fusion Technol. 19, 131(1991).

[14] H. Ji et al., Rev. Sci. Instrum., 62, 2326(1991).

[15] A.F. Almagri, et al., Phys. Fluids, B4, 4080(1992).

[16] P.W. Terry and P.H. Diamond, Phys. Fluids, B2, 1128(1990). 


\section{FIGURES}

FIG. 1. Waveforms of toroidal flux $\Phi_{t}$, pinch parameter $\Theta$, reversal parameter $F$, ion saturation current $J_{s a t}$ measured by the triple probe at $r / a=0.98$, and voltage across toroidal gap in the shell $V_{\mathrm{tg}}$ during two sawtooth oscillations.

FIG. 2. Ensemble-averaged fluctuation amplitudes, coherence, and phase shift during one sawtooth cycle, measured at $r / a-0.90$. The toroidal gap voltage, $V_{\mathrm{tg}}$, marks the timing of the sawtooth crash.

FIG. 3. Ensemble-averaged MHD dynamo electric fields and local parallel current density during one sawtooth crash, measured at $r / a=0.90$.

FIG. 4. Comparison of MHD dynamo electric field $<\tilde{\mathbf{E}}_{\perp} \cdot \tilde{\mathbf{b}}_{\perp}>$ to (a) $\eta j_{\|}$(b) $\eta j_{\|}-E_{\|}$during one sawtooth crash. Rapid oscillations in $\left\langle\tilde{\mathbf{E}}_{\perp} \cdot \tilde{\mathbf{b}}_{\perp}\right\rangle$ indicate experimental uncertainty.

FIG. 5. Edge radial profiles of MHD dynamo electric field $\left\langle\tilde{\mathbf{E}}_{\perp} \cdot \tilde{\mathbf{b}}_{\perp}>\right.$ and $\eta j_{\|}-E_{\|}$between and during the sawtooth crashes. The shaded region (blackened region) indicates the measurement uncertainty of $<\widetilde{\mathbf{E}}_{\perp} \cdot \tilde{\mathbf{b}}_{\perp}>\left(\eta j_{\|}-E_{\|}\right)$. 


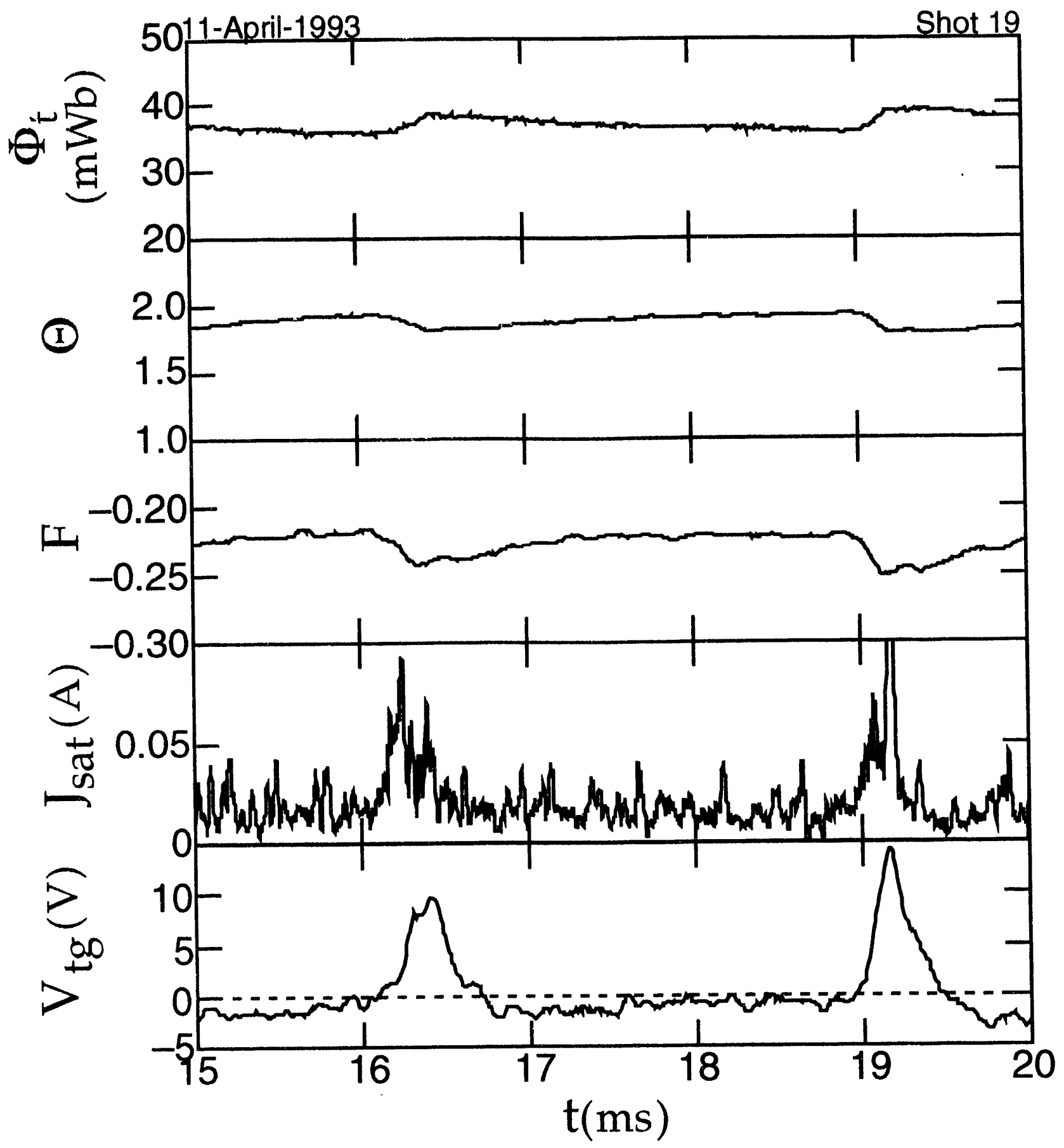

"igure] 


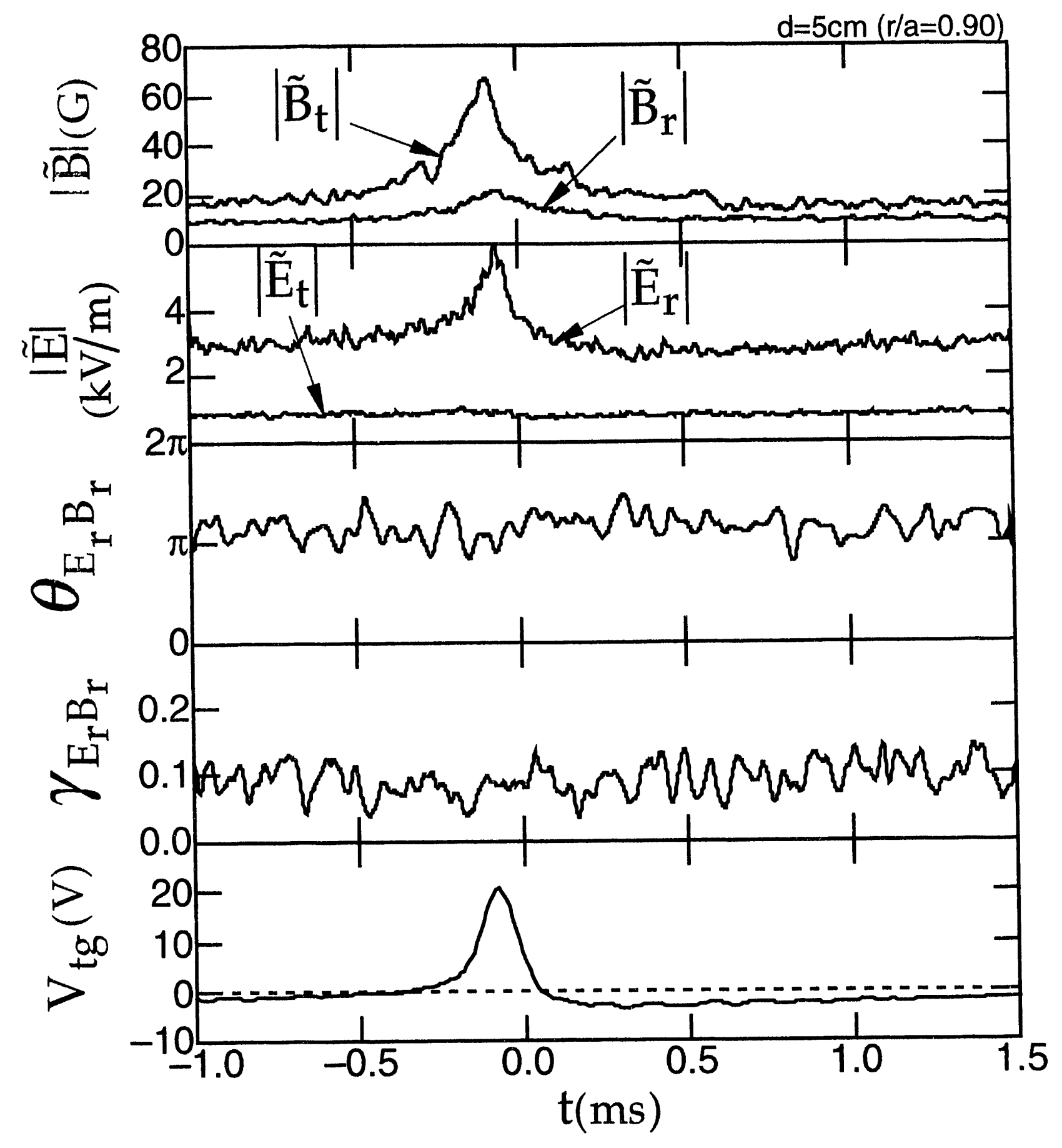

Figure 2 


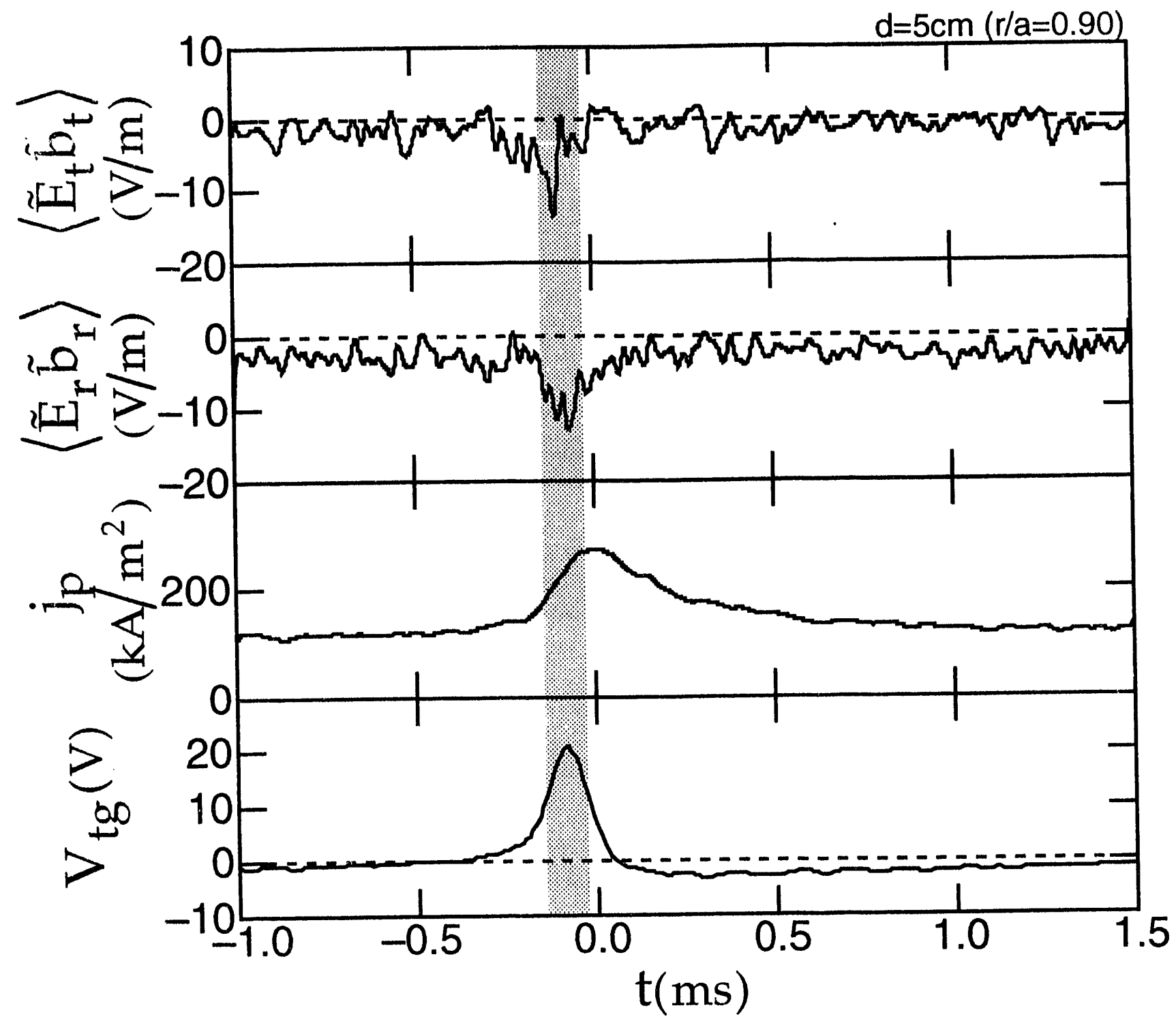

Figure 3 


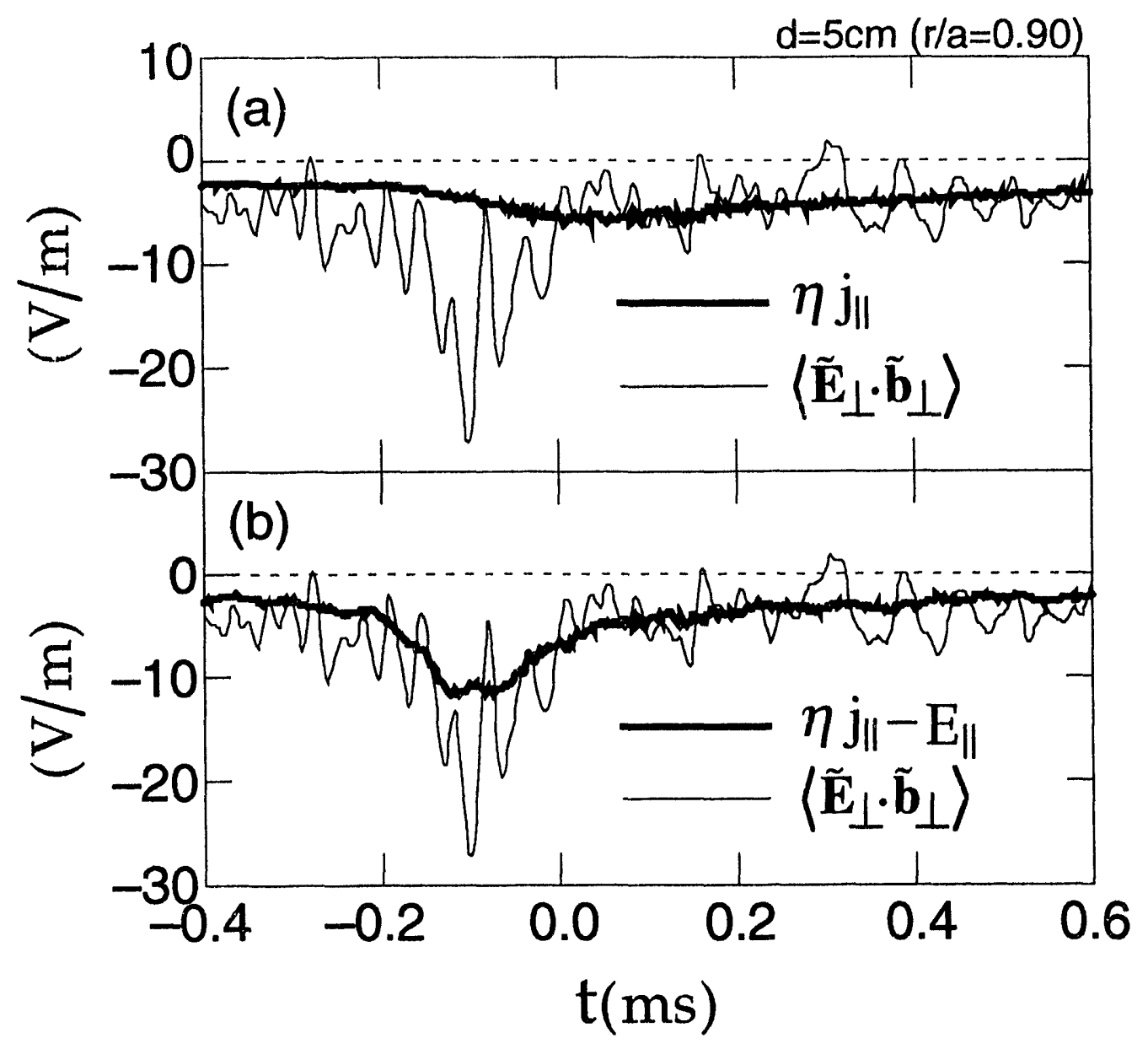

Figure 4 


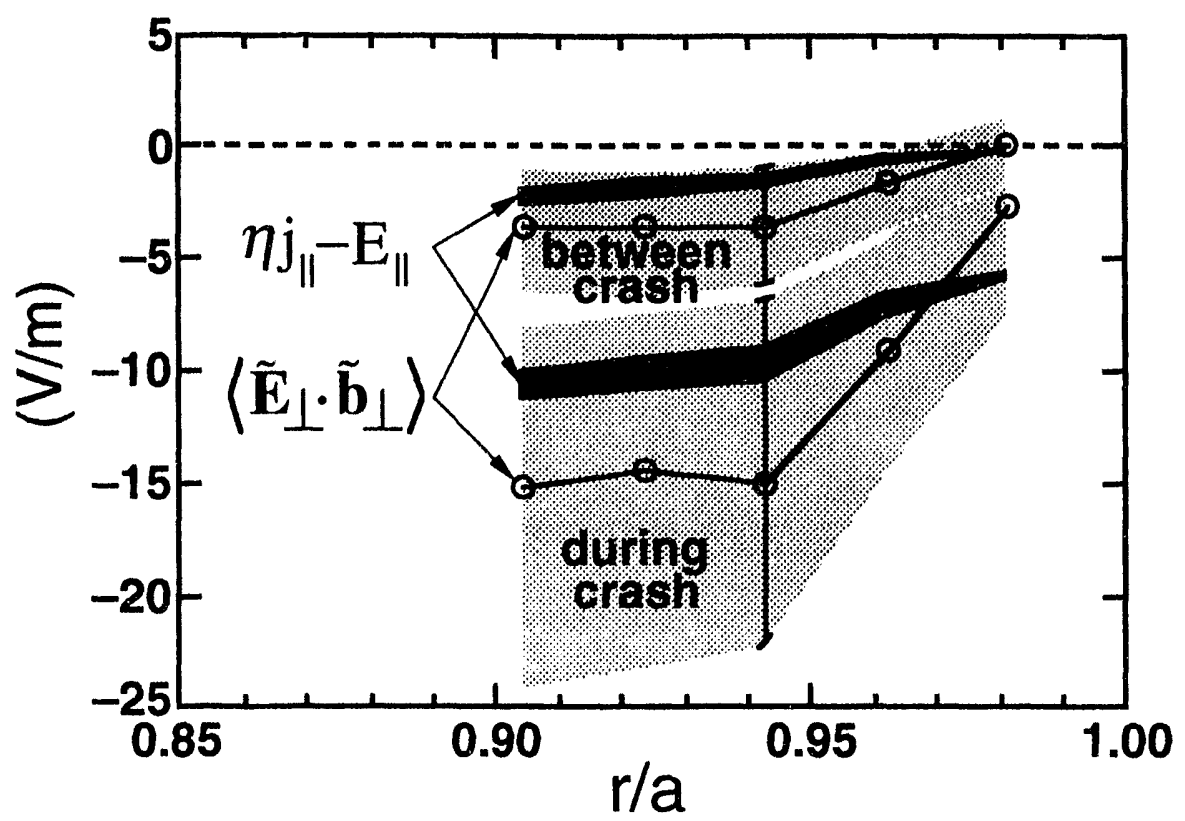

Pinures 


\section{EXTERNAL DISTRIBUTION IN ADDITION TO UC-20}

S.N. Rasband, Brigham Young University

T. Dolan, EG\&G Idaho, Inc.

R.A. Moyer, General Atomics

J.B. Taylor, Institute for Fusion Studies, The University of Texas at Austin

E. Uchimoto, University of Montana

F.W. Perkins, PPPL

O. Ishihara, Texas Technical University

M.A. Abdou, University of California, Los Angeles

R.W. Conn, University of California, Los Angeles

P.E. Vandenplas, Association Euratom-Etat Belge, Belgium

Centro Brasileiro de Pesquisas Firicas, Brazil

P. Sakanaka, Institute de Fisica-Unicamp, Brazil

Mme. Monique Bex, GANIL, France

J. Radet, CEN/CADARACHE, France

University of Ioannina, Greece

S. Ortolani, Istituto Gas Ionizzati, EURATON-ENEA-CNR Association, Italy

R. Andreani, Associazione EURATOM-ENEA sulla Fusione, Italy

Plasma section, Energy Fundamentals Division Electrotechnical Laboratory, Japan

Y. Kondoh, Gunma University, Kiryu, Gunma, Japan

H. Toyama, University of Tokyo, Japan

Z. Ypsjoda. University of Tokyo, Japan

FOM-Instituut voor Plasmafysica "Rijnhuizen," The Netherlands

Z. Ning, Academia Sinica, Peoples Republic of China

P. Yang, Shandong University, Peoples Republic of China

S. Zhu, University of Science \& Technology of China, People's Republic of China

I.N. Bogatu, Institute of Atomic Physics, Romania

M.J. Alport, University of Natal, Durban, South Africa

R. Storer, The Flinders University of South Australia, South Australia

B. Lehnert, Royal Institute of Technology, Sweden

Librarian, CRPP, Ecole Polytechnique Federale de Lausanne, Switzerland

B. Alper, Culham Laboratory, UK

A. Newton, UK

2 for Chicago Operations Office

5 for individuals in Washington Offices

INTERNAL DISTRIBUTION IN ADDITION TO UC-20

80 for local group and file 

